\title{
In-hospital complications following primary total hip and knee arthroplasty in octogenarian and nonagenarian patients
}

\author{
Shashi K. Nanjayan • Girish N. Swamy • \\ Sunil Yellu • Sachin Yallappa · Tarek Abuzakuk • \\ Robert Straw
}

Received: 10 January 2013/Accepted: 22 July 2013/Published online: 29 August 2013

(C) The Author(s) 2013. This article is published with open access at Springerlink.com

\begin{abstract}
Background As life expectancy of patients increases, more elderly patients are undergoing primary total hip arthroplasty (THA) and total knee arthroplasty (TKA). There is a general perception of increased risk of complications in elderly patients. Our objective was to analyse the incidence of in-hospital medical and surgical complications following THA and TKA in octogenarian and nonagenarians.

Materials and methods This was a prospective review of 202 consecutive patients aged more than 80 years who underwent total hip and total knee arthroplasty (101 THA, 101 TKA) over an 18-month period. In this single-centre observational study, collected data included patient demographics, American Society of Anethesiologists (ASA) grade, length of hospital stay and peri-operative medical and surgical complications during their hospital stay.

Results Median age of patients was 83 years. Median ASA grade was 3. Mean length of hospital stay was 7.5 days. There were 14 major systemic complications in the THA group and 13 in the TKA group. While 1 major local complication occurred in each group, there were 6 minor local complications in THA and 7 in the TKA group. All the complications occurred within 5 post-operative days. There was no in-hospital mortality.

Conclusion In our study, we found that the incidence of peri-operative medical and surgical complications is higher in those over 80 years, compared to the published literature in patients of all age groups undergoing THA and TKA. Awareness of a higher incidence of major systemic
\end{abstract}

S. K. Nanjayan $(\varangle) \cdot$ G. N. Swamy $\cdot$ S. Yellu · S. Yallappa

T. Abuzakuk · R. Straw

Royal Derby Hospital, Uttoxeter New Road,

Derby DE22 3NE, UK

e-mail: shashikumartn@gmail.com complications should alert the treating surgeon to carry out comprehensive peri-operative management in this subset of patients, which could lead to better outcomes.

Keywords In-hospital complications .

Octogenarian $\cdot$ Nonagenarian $\cdot$ Arthroplasty

\section{Introduction}

Primary total knee arthroplasty (TKA) and primary total hip arthroplasty (THA) are the most commonly performed elective orthopaedic operations in the United Kingdom. The average age of patients undergoing TKA is 67.4 years, and those undergoing THA is 67.2 years [1]. The life expectancy at birth for the UK population was 78.2 years for males and 82.3 years for females between 2008-2010 [2].

As the life expectancy of patients is increasing, more elderly patients are undergoing THA and TKA due to increasing incidence of osteoarthritis in this age group. The recent advances in health care have enabled these patients to undergo major procedures like TKA and THA, helping them to pursue more active lifestyles. There is a general perception of increased risk of 'in-hospital' complications in elderly patients undergoing TKA or THA. This could be due to their general health status and their associated medical co-morbidities. Advancing age is reported to be a significant risk factor in the incidence of in-hospital complications [3].

The objective of this study was to analyse the incidence of in-hospital peri-operative medical and surgical complications following THA and TKA in patients with advanced age, that is, in octogenarians and nonagenarians, and to compare the same with the published literature. 


\section{Materials and methods}

A prospective review of 202 consecutive patients aged more than 80 years, who underwent total hip and total knee arthroplasty (101 THA, 101 TKA) during an 18-month period was carried out.

The inclusion criterion was:

1. All patients older than 80 years of age undergoing unilateral primary THA or primary TKA

Exclusion criteria were:

1. Patients undergoing revision THA or revision TKA

2. Patients undergoing unicompartmental TKA

3. Patients under the age of 80 years undergoing primary THA and primary TKA

No enrolled patients were excluded during the study.

All the patients gave informed consent prior to being included in the study. All the operations were unilateral and primary arthroplasties. In this single-centre observational study, collected data included patient demographics, American Society of Anethesiologists (ASA) grade, length of hospital stay and peri-operative medical and surgical complications during their hospital stay. All these patients underwent pre-operative assessment, a few weeks prior to the surgery, led by the surgeon and the anaesthetist. If any medical issues were identified, they were optimised prior to the surgery. Unless contraindicated or an attempt failed, all the patients received spinal anaesthesia with an additional regional block. All operations were performed in dedicated orthopaedic operating theatres, which have a laminar air flow system. Trainees performed $19.3 \%(n=39)$ of the operations under the supervision of consultants, while the consultant surgeons performed $80.7 \%(n=163)$ of the operations.

Forty-one of the THAs were performed using a posterior approach, and 60 with a modified anterolateral approach. Different surgical approaches for THA were used according to the surgeon's preference. Ninety-nine were cemented THA and 2 were hybrid THA.

All TKAs were performed with the use of a tourniquet and medial parapatellar approach. All TKA implants were metal backed and fixation was cemented. We did not use any wound drains for the total hip arthroplasty. Eightyseven patients had drains for TKA, which were removed routinely at $24 \mathrm{~h}$. Placement of drains following TKA was according to the surgeon's preference.

A multimodal method was used for venous thromboembolism (VTE) prophylaxis, using TED stockings, foot pumps and aspirin or low molecular weight heparin (enoxaparin $40 \mathrm{mg}$ subcutaneously, once daily) for 6 weeks post-operatively. Intravenous antibiotics were given at induction of anaesthesia, with two further doses in the next $24 \mathrm{~h}$. All patients were encouraged to mobilise the same day or the day after surgery.

Check radiographs, full blood count, urea and electrolyte were tested the following day, unless the clinical condition of the patient dictated earlier investigation.

All the complications that occurred during the patients' stay in hospital were recorded in the database.

We analysed the age, gender, height, weight, BMI, type of anaesthesia, ASA grade, surgeon's grade and type of thromboprophylaxis and drop in post-operative haemoglobin as risk factors for occurrence of complications.

The complications were classified as major and minor systemic complications and major and minor local complications as used in studies by Parvizi et al. [3] and Pulido et al. [4].

Statistical analysis was done using R Software. Univariate regression analysis was performed on all risk factors for complications. Multiple logistic regression analysis was performed on all variables that were significant in univariate analysis. For the univariate analysis, a $p$ value of $<0.1$ was considered significant. For all other tests, a $p$ value of $<0.05$ was considered significant.

\section{Results}

The median age of patients was 83 years (IQR 80-95 years). There were 150 patients aged between 80 and 85 years, 39 patients between 86 and 90 years and 13 aged more than 90 years. There were 126 female patients and 76 male.

The most common ASA grade was either ASA 2 $(n=111)$ or ASA $3(n=85)$. The median BMI was 28 (IQR 25-31). Median height was $162 \mathrm{~cm}$ (IQR 156-170 cm).

Median weight was $75 \mathrm{~kg}$ (IQR 65-86 kg). Mean length of hospital stay was 7.5 days. Nearly $24 \%(n=48)$ of patients required blood transfusion.

We found 14 major systemic complications in the THA group and 13 major systemic complications in the TKA group. There were 16 minor systemic complications in THA and 17 minor systemic complications in TKA. One major local complication was found in both THA and TKA groups (Table 1). All complications occurred within 5 post-operative days.

We did not have any in-hospital mortality in our cohort of patients.

Univariate regression analysis revealed that significant factors for complications were 'length of stay in the hospital' and reception of 'blood transfusion' (Table 2).

Multivariate logistic regression using the Akaike information criterion (AIC) revealed that patients who stayed in the hospital for more than 5 days $(p<0.00001)$ and those 
Table 1 Incidence of complications

\begin{tabular}{|c|c|c|}
\hline Major systemic complications & $\begin{array}{l}\text { ТНA } \\
(n=101)\end{array}$ & $\begin{array}{l}\text { TKA } \\
(n=101)\end{array}$ \\
\hline \multicolumn{3}{|l|}{ Cardiovascular } \\
\hline Pulmonary embolism & 0 & 2 \\
\hline Atrial fibrillation & 3 & 4 \\
\hline Myocardial infarction & 1 & 1 \\
\hline Pulmonary oedema & 1 & 0 \\
\hline \multicolumn{3}{|l|}{ Arrhythmia } \\
\hline Deep vein thrombosis & 0 & 0 \\
\hline \multicolumn{3}{|l|}{ Neurological } \\
\hline Stroke & 0 & 1 \\
\hline \multicolumn{3}{|l|}{ Pulmonary } \\
\hline Respiratory arrest (opioid induced) & 1 & 0 \\
\hline Pneumonia & 4 & 3 \\
\hline \multicolumn{3}{|l|}{ Gastrointestinal } \\
\hline Gastrointestinal bleed & 2 & 0 \\
\hline Altered liver function & 1 & 0 \\
\hline Renal/fluid and electrolyte imbalance & & \\
\hline Acute kidney injury & 1 & 1 \\
\hline Minor systemic complications & & \\
\hline Cardiovascular & & \\
\hline Angina & & 0 \\
\hline Sinus tachycardia & & 1 \\
\hline Hypotension & & 1 \\
\hline Neurological & & \\
\hline Confusion/delirium & & 5 \\
\hline Urinary & & \\
\hline Urinary tract infection & & 1 \\
\hline Urinary retention & & 1 \\
\hline Fluid and electrolyte imbalance & & \\
\hline Hypokalemia & & 3 \\
\hline Hyponatremia & & 3 \\
\hline Gastrointestinal & & \\
\hline Diarrhoea (Clostridium difficile-negative) & & 2 \\
\hline General & & \\
\hline Fall in the hospital & & 1 \\
\hline Vasovagal episode/unresponsiveness & & 1 \\
\hline In-hospital mortality & & 0 \\
\hline Local complications & $\begin{array}{l}\text { THA } \\
(n=101)\end{array}$ & $\begin{array}{l}\text { TKA } \\
(n=101)\end{array}$ \\
\hline Major & & \\
\hline Dislocation & 1 & 0 \\
\hline Peripheral nerve injury (foot drop) & 0 & 1 \\
\hline Vascular injuries & 0 & 0 \\
\hline Minor & & \\
\hline Skin tear & 1 & 0 \\
\hline Wound haematoma & 1 & 1 \\
\hline Wound leakage & 1 & 2 \\
\hline Superficial wound infection & 1 & 1 \\
\hline Blisters & 1 & 2 \\
\hline Cellulitis & 1 & 2 \\
\hline
\end{tabular}

Table 2 Univariate analysis of variables

\begin{tabular}{llc}
\hline Variable & Statistic value & $p^{\prime}$ value \\
\hline TKA vs THA & 0.19 & 0.66 \\
Age & 1.90 & 0.31 \\
Gender & 2.20 & 0.14 \\
Height & $1.21(z$ value $)$ & 0.23 \\
Weight & $0.13(z$ value $)$ & 0.90 \\
BMI & $0.89(z$ value $)$ & 0.37 \\
Surgeon grade (trainee vs consultant $)$ & 0.10 & 0.75 \\
Type of anaesthesia & 3.23 & 0.52 \\
Type of DVT prophylaxis & 2.97 & 0.81 \\
ASA grade & 1.29 & 0.73 \\
Length of hospital stay & 5.53 & $<0.00001$ \\
Transfusion & 17.32 & $<0.012$ \\
Drop in haemoglobin $(<4 \mathrm{vs}>4 \mathrm{mg} / \mathrm{dl})$ & 4.02 & 0.13 \\
\hline
\end{tabular}

who received blood transfusion had a significant $p$ value $(p<0.012)$. Likelihood of suffering a complication was slightly higher in these groups (LOS - odds ratio 3.12 and transfusion - odds ratio 3.38). This probably reflects the fact that a patient who developed a complication probably stayed a few days more in the hospital and was more likely to receive a blood transfusion.

\section{Discussion}

With increasing advances and standards in the peri-operative care of patients, in-hospital complications are expected to be low. But, in older patients, especially octogenarians and nonagenarians, there is an increased incidence of complications [4-7].

Clement et al. [5] in their prospective study comparing a selected group of patients aged more than or equal to 80 years and a control group aged between 65 and 74 years, reported increased medical complications in patients older than 80 years. The older group had a longer hospital stay for both THA and TKA.

In a larger series of 15,383 patients undergoing total joint arthroplasties, Pulido et al. [4] reported a major systemic complication in $2.02 \%$ of primary THA and $4.91 \%$ of primary TKA, as well as minor systemic complications in $3.17 \%$ of primary THA and $3.40 \%$ of primary TKA. We found a much higher incidence of major complications (14\% in the THA group and $13 \%$ in the TKA group). The incidences of minor systemic complications were also found to be higher in our group (16\% in THA and $17 \%$ in TKA).

They reported the incidence of major local complications at $0.48 \%$ for primary THA and $0.83 \%$ for primary TKA, whereas we found $1 \%$ incidence in our cohort. 
Minor local complications were also increased in our cohort; $6 \%$ in THA and $7 \%$ in TKA when compared to $1.95-2.54 \%$ in their series. Incidences of these complications are more in our group. Our cohort was of older patients with a median age of 83 years, whereas the mean age in the Pulido et al. [4] series was 61 years in males and 65 years in females.

Wurtz et al. [8] reported $27 \%(n=11)$ medical-related complications and $15 \%(n=6)$ hip-related complications in the octogenarian population undergoing THA. We found a similar major systemic complication rate in our cohort.

The most common complication observed in our study was an episode of post-operative cognitive dysfunction (POCD). There were 9 cases of POCD in our study (4 in THA and 5 in TKA). Investigation carried out in these patients revealed urinary tract infection in one patient and hyponatremia in another patient, which resolved after instituting the appropriate treatment. In the remaining 7 patients, no identifiable cause was found, and it resolved prior to discharge. Confusion in hospitalised elderly patients is multifactorial and its incidence increases with increasing age [9]. Older age, peri-operative use of narcotics, polypharmacy, acute pain, urinary catheter, addition of 3 or more medications in 24-48 h, anaemia, fluid and electrolyte imbalance, greater surgical blood loss and intraoperative transfusion have been reported to be triggers of post-operative cognitive dysfunction [10].

The incidence of POCD in elderly patients has been reported to be between 5 and $15 \%$ [11], and increases to about $25 \%$ in older patients in the immediate post-operative days [12]. The decreasing trend in the incidence of POCD is probably because of the increasing standards of peri-operative care.

Larger series have reported clear age- and sex-specific frequencies of myocardial infarction (MI) in the perioperative period following THA and TKA. The increases in incidence of adverse events like MI, pulmonary embolism (PE), DVT and death was slightly more in patients aged more than 80 when compared to younger patients [13, 14]. Two cases of PE in our patients were, after TKA, proven on a computed tomographic pulmonary angiogram (CTPA). One case of MI in each THA and TKA were treated according to the hospital cardiology guidelines.

One study has shown that incidence of DVT did not differ significantly with age, sex or type of operation [13]. There was no incidence of DVT in our patients, although 7 patients (5 TKA, 2 THA) underwent DVT study (vascular ultrasound) with suspected DVT, but none of them were proven positive.

Atrial fibrillation (AF) was observed in $3 \%$ of THA and $4 \%$ of TKA in our series. A larger series [4], with a mean age between 61 years for males and 65 years for females reported an incidence of $0.4 \%$ in THA and $0.5 \%$ in TKA.
Increasing age is a known, independent variable in the incidence of AF [15]. The findings in our series are similar.

ASA grade as a comorbidity index is validated in hip fracture surgery [16] and it is a useful indicator of perioperative patient health status [17]. We found that the ASA grade was not a key predictor of occurrence of complication in this cohort of patients.

Patel et al. [18] showed that morbid obesity was an independent risk factor for prolonged wound drainage following joint arthroplasties, irrespective of type of VTE prophylaxis. We had two cases of wound haematoma (1 THA, 1 TKA) and 3 cases of wound leakage (1 THA, 2 TKA). The mean BMI of our patients was 28 and our usual DVT prophylaxis was multimodal (aspirin $150 \mathrm{mg}+$ TED stockings + peri-operative foot pump). Neither BMI nor type of DVT prophylaxis was a significant variable for occurrence of in-hospital complications in our study.

Acute vascular complications are very rare following THA and TKA [19]. Although risk of arterial calcification is slightly higher in older patients, rendering them vulnerable to arterial injuries, fortunately, we did not have any such complications.

In-hospital mortality has been shown increase at least ninefold in patients older than 85 years, when compared to patients aged between 45 and 64 years [20]. ASA grade is a significantly related to post-operative death [17]. We did not have any mortality in our cohort despite the mean age of 83 years. Nevertheless, this cannot undermine the importance of careful and meticulous planning of perioperative care needed for octogenarians, as THA and TKA is increasingly performed in this population.

Incidence of these complications was slightly more in our group when compared to the largest series published in the literature [4], but our cohort was of older patients with a median age of 83 years, whereas the mean age in the $\mathrm{Pu}-$ lido et al. [4] series was 61 years in males and 65 years in females.

A limitation of our series was that our cohort was relatively small, keeping in mind the frequency of THA and TKA.

The option of recording 'in-hospital complication' in the National Joint Registers should be considered. This would allow better capture of the data on a national database, which would then allow better understanding and thus better standards of care to our patients. The analysis of more data can be used to obtain better informed consent from this cohort of patients.

In conclusion, our study found that the incidence of perioperative medical and surgical complications is more common in those over 80 years old compared to the published literature in patients of all age groups undergoing total hip and knee arthroplasty. Awareness of the higher incidence of major systemic complications in this subset of patients 
should alert the treating surgeon to carry out a comprehensive pre-operative evaluation to optimise the co-existing medical condition. Further, vigilant post-operative management of these patients could lead to better outcomes.

\section{Conflict of interest None.}

Ethical standards statement This study conforms to the Declaration of Helsinki. The Institutional Review Board approved the study, and all the patients provided informed consent prior to their enrollment.

Open Access This article is distributed under the terms of the Creative Commons Attribution License which permits any use, distribution, and reproduction in any medium, provided the original author(s) and the source are credited.

\section{References}

1. National Joint Registry for England and Wales, 9th annual report. http://www.njrcentre.org.uk

2. http://www.ons.gov.uk/ons/dcp171778_238743.pdf

3. Parvizi J, Mui A, Purtill JJ, Sharkey PF, Hozack WJ, Rothman RH (2007) Total joint arthroplasty: when do fatal or near-fatal complications occur? J Bone Joint Surg Am 89(1):27-32

4. Pulido L, Parvizi J, Macgibeny M et al (2008) In hospital complications after total joint arthroplasty. J Arthroplasty 23(6 Suppl 1):139-145

5. Clement ND, MacDonald D, Howie CR, Biant LC (2011) The outcome of primary total hip and knee arthroplasty in patients aged 80 years or more. J Bone Joint Surg Br 93(9):1265-1270

6. SooHoo NF, Lieberman JR, Ko CY, Zingmond DS (2006) Factors predicting complication rates following total knee replacement. J Bone Joint Surg Am 88(3):480-485

7. Gill GS, Mills D, Joshi AB (2003) Mortality following primary total knee arthroplasty. J Bone Joint Surg Am 85-A(3):432-435

8. Wurtz LD, Feinberg JR, Capello WN, Meldrum R, Kay PJ (2003) Elective primary total hip arthroplasty in octogenarians. J Gerontol A Biol Sci Med Sci 58(5):M468-M471
9. Belmar CJ, Barth P, Lonner JH, Lotke PA (1999) Total knee arthroplasty in patients 90 years of age and older. J Arthroplasty 14(8):911-914

10. Deiner S, Silverstein JH (2009) Postoperative delirium and cognitive dysfunction. Br J Anaesth 103(Suppl 1):i41-i46

11. Bekker AY, Weeks EJ (2003) Cognitive function after anaesthesia in the elderly. Best Pract Res Clin Anaesthesiol 17(2): 259-272

12. Abildstrom H, Rasmussen LS, Rentowl P et al (2000) Cognitive dysfunction 1-2 years after non-cardiac surgery in the elderly. ISPOCD group. International Study of Post-Operative Cognitive Dysfunction. Acta Anaesthesiol Scand 44(10):1246-1251

13. Mantilla CB, Horlocker TT, Schroeder DR, Berry DJ, Brown DL (2002) Frequency of myocardial infarction, pulmonary embolism, deep venous thrombosis, and death following primary hip or knee arthroplasty. Anesthesiology 96(5):1140-1146

14. Austin L, Pulido L, Ropiak R, Porat M, Parvizi J, Rothman RH (2008) Hypoxemia after total joint arthroplasty: a problem on the rise. J Arthroplasty 23(7):1016-1021

15. Kahn RL, Hargett MJ, Urquhart B, Sharrock NE, Peterson MG (1993) Supraventricular tachyarrhythmias during total joint arthroplasty. Incidence and risk. Clin Orthop Relat Res(296): 265-269

16. Bjorgul K, Novicoff WM, Saleh KJ (2010) American Society of Anesthesiologist Physical Status score may be used as a comorbidity index in hip fracture surgery. J Arthroplast 25(6 Suppl): 134-137

17. Rauh MA, Krackow KA (2004) In-hospital deaths following elective total joint arthroplasty. Orthopedics 27(4):407-411

18. Patel VP, Walsh M, Sehgal B, Preston C, DeWal H, Di Cesare PE (2007) Factors associated with prolonged wound drainage after primary total hip and knee arthroplasty. J Bone Joint Surg Am 89(1):33-38

19. Calligaro KD, Dougherty MJ, Ryan S, Booth RE (2003) Acute arterial complications associated with total hip and knee arthroplasty. J Vasc Surg 38(6):1170-1177

20. Memtsoudis SG, Della Valle AG, Besculides MC, Esposito M, Koulouvaris P, Salvati EA (2010) Risk factors for perioperative mortality after lower extremity arthroplasty: a population-based study of 6,901,324 patient discharges. J Arthroplast 25(1):19-26 